\title{
ANALGESIA, SEDAÇÃO E BLOQUEIO NEUROMUSCULAR EM UTI
}

\author{
ANALGESIA, SEDA TIONAND NEUROMUSCULAR BLOCKADE INTHE ICU
}

Alexandre Slullitel \& Angela M. Sousa

\begin{abstract}
${ }^{1}$ Assistente da Divisão de Anestesiologia do Instituto do Coração (InCor) Hospital das Clínicas da Faculdade de Medicina de São Paulo; ${ }^{2}$ Assistentes da Disciplina de Anestesiologia do Hospital das Clínicas da Faculdade de Medicina de Ribeirão Preto — Universidade de São Paulo.

Correspondencia: Disciplina de Terapia Intensiva, Departamento de Cirurgia, Ortopedia e Traumatologia da Faculdade de Medicina de Ribeirão Preto da Universidade de São Paulo - Hospital das Clínicas - 9 Andar - Campus Universitário CEP: $14048-900$ - Ribeirão Preto-SP Fone: (016) 633-0836 ou 602-2593. E-mail : slullite@usp.br. E-mail: abasile@.fmrp.usp.br
\end{abstract}

SLULLITEL A \& SOUSA AM. Analgesia, sedação e bloqueio neuromuscular em UTI. Medicina, Ribeirão Preto, 31: 507-516, out./dez. 1998

RESUMO: A dor é um sintoma freqüentemente associado ao paciente crítico, $\mathrm{O}$ tratamento adequado desta condição está relacionado não apenas aos processos de humanização na UTI como também a modificar o prognóstico e reduzir os custos hospitalares. São apresentados métodos de avaliação da intensidade da dor e técnicas de tratamento, desenvolvidos pela equipe atuante na UTI. Exposição a um ambiente com grande sobrecarga de estímulos sensitivos, dolorosos, ruído, aspiração traqueal e a privação de sono pode requerer o uso de drogas para controlar a ansiedade e a inquietude. Além disso, algumas situações clínicas, tais como a ventilação mecânica, podem não dispensar a sedação para lograrem sucesso. A importância e as indicações clínicas do uso de relaxantes musculares são revistas.

UNITERMOS: Analgesia. Bloqueios Neuromusculares. Sedação Consciente. Unidades de Terapia Intensiva.

\section{INTRODUÇÃO}

A dor é definida pela IASP (Associação Internacional para o Estudo da Dor), como "sensação desagradável e experiência emocional, associada à lesão tecidual real ou potencial, ou descrita em termos relacionados à lesão".

Dor aguda é definida por Bonica ${ }^{(1)}$ como uma “complexa constelação de experiências desagradáveis, perceptuais, psicológicas, emocionais e respostas comportamentais causadas por estimulação nociva”. Esta é invariavelmente produzida por lesão e/ou doença na pele, estruturas somáticas profundas ou vísceras, ou função anormal de músculos ou vísceras, na ausência de lesão.

Dor crônica é definida como aquela que "persiste por mais de um mês além do curso normal ou que seja associada a processo patológico crônico, que cause dor contínua ou recorrente a intervalos de meses ou anos"(2).

A intensidade, à qual a dor parece insuportável, varia não somente de individuo a indivíduo, grau de ansiedade, mas, também, de acordo com a cultura em que o indivíduo é educado.

No século 19. ocorreu o início do estudo da fisiologia como ciência e essa era foi iniciada, em parte, por Bell \& Magendie, que em 1811 e 1827, demostraram, em experimentos em animais, que a função das raízes dorsais é sensitiva e a das raízes ventrais é motora ${ }^{(1)}$.

\section{ANATOMIA}

Admite-se que as mensagens nociceptivas se- 
jam geradas em terminações livres, que são geralmente muito numerosas, da ordem de $200 / \mathrm{cm}^{2}$ e muito freqüentemente solidárias a vênulas e arterío- las. Esses nociceptores são terminações livres de fibras arnielinizadas (fibras C) e fibras mielinizadas de pequeno diâmetro (fibras $\mathrm{A} \delta)^{(1)}$. corpo celular desses neurônios localiza-se no gânglio da raiz dorsal e é, na maioria das vezes, bipolar, com um braço na periferia e outro no corno dorsal da medula. Denomina- se primário esse conjunto aferente.

Os axônios do aferente primário terminam na pele, tecido subcutâneo, periósteo, articulações, músculos e vísceras. Alguns respondem a estímulos mecânicos (mecanoceptores), estímulos mecânicos e térmicos (mecanotermo nociceptores) e estímulos térmicos, mecânicos e químicos (receptores polimodais). Os nociceptores são caracterizados por seu campo restrito e sua habilidade de responder unicamente ou diferencialmente aos estímulos nocivos ${ }^{(1)}$.

Após penetrar na medula espinhal, muitas raízes dorsais bifurcam em $Y$ e divide-se em um longo ramo ascendente e um pequeno ramo descendente, $\mathrm{e}$ fazem conexão com neurônios de segunda ordem em um nível diferente daquele em que ocorre à entrada da fibra no corno dorsal. A maio ria dos aferentes primários terminam no lado ipsilateral, mas uma percentagem variável cruza a medula e termina rio lado contralateral. $\mathrm{Na}$ medula espinhal, o aferente primário faz sinapse com neurônios, em diversos níveis, divididos em lâminas de $1 \mathrm{a}$ $\mathrm{X}$. As fibras $\mathrm{C}$, condutoras de estímulos dolorosos, em baixa velocidade, terminam principal mente em lâminas 1 , II e $\mathrm{V}$ e as fibras $\mathrm{A} \delta$ mielinizando-se, termiliam em lâminas $\mathrm{V}$ e $\mathrm{X}^{(1)}$.

A informação nociceptiva é transmitida ao cé- rebro através de vias nervosas, localizadas rio quadrante anterolateral da medula espinhal. O trato espinotalârnico, o espinorreticular e o espinome sencefálico são considerados como as vias ascendentes mais importantes de condução de impulsos nociceptivos em humanos e primatas. A parte lateral do trato espinotalâmico é denominada neoespinotalâmico e a sua parte medial mais o trato espinorreticular e o espinomesencefálico correspondem ao pa leoespinotalâmico. O trato neoespinotalâmico projeta-se ao núcleo ventro-posterolateral do tálamo e daí ao córtex somatossensorial. O sistema paleoespino talâmico projeta-se para a formação reticular, substância cinzenta periaquedutal, hipotálamo, núcleo talârnico medial e intralaminar ${ }^{(1)}$.. Essas fibras se conectam com estruturas límbicas e projetam-se difusamente no córtex cerebral. Após lesão desses tratos, a sensação dolorosa é abolida do lado contralateral (Figura 1).

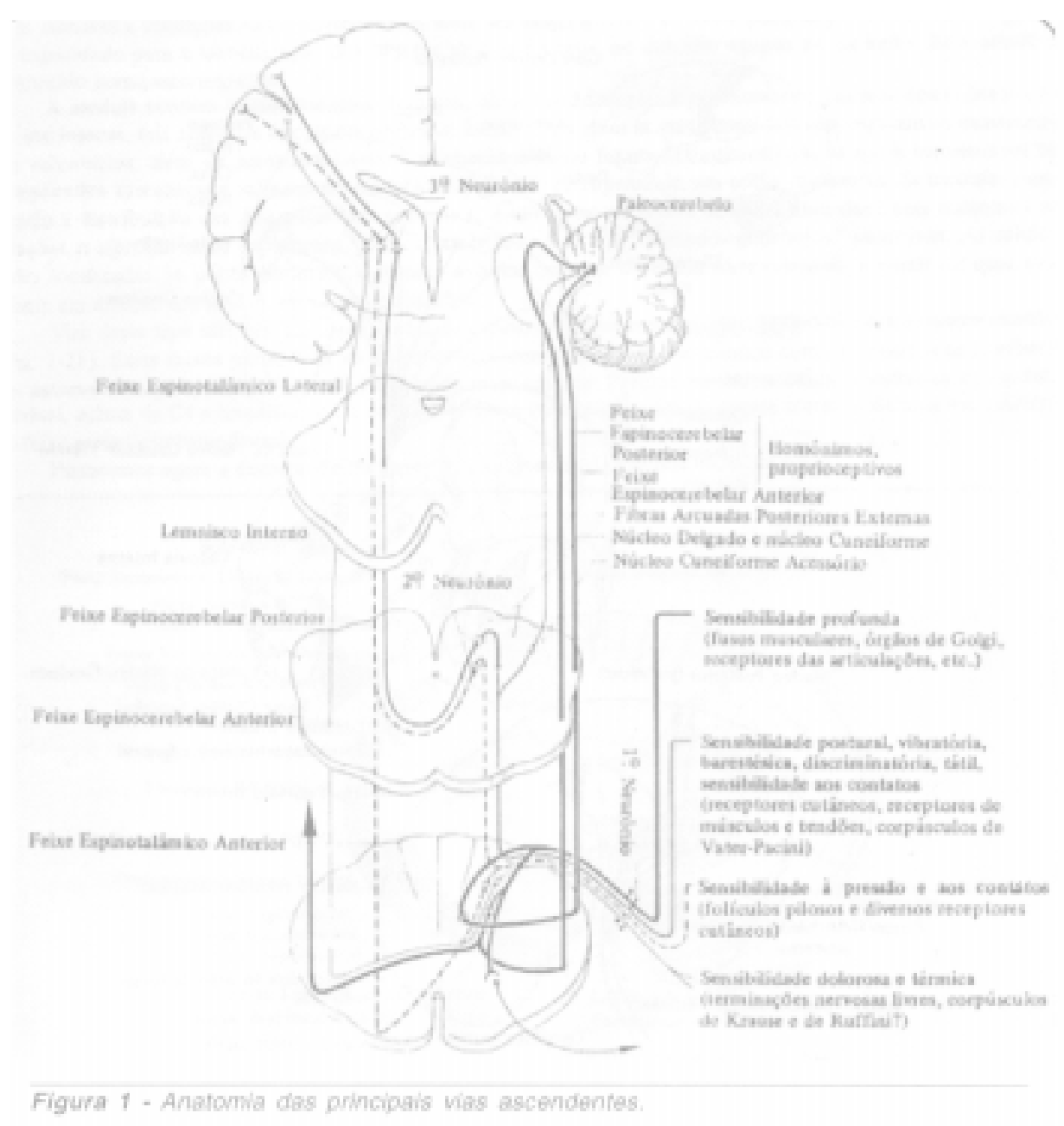




\section{MONITORIZAÇÃO DA DOR}

É fundamental assegurar analgesia suficiente, antes e durante a sedação, e prover hipnose, antes e durante o bloqueio neuromuscular ${ }^{(3)}$.

Um dos aspectos mais importantes da analgesia é a determinação individual da dor. A primeira etapa, na tentativa de estabelecer o controle eficaz da dor, é o desenvolvimento de um método prático para sua avaliação quali e quantitativa, tornando - a objeto de discussões durante as visitas. Particularmente, a es cala analógica e visual (EAV) tem ampla utilização em clínica e pesquisas, onde é necessário um índice de dor ao qual se pode atribuir um valor numérico. Consiste em uma linha horizontal ou vertical de 10 $\mathrm{cm}$, com as duas extremidades rotuladas como "sem dor" e "pior dor imaginável". Solicita-se ao paciente que marque, na linha de $10 \mathrm{~cm}$, o ponto que corres potide ao nível de intensidade da dor que está sentindo. A distância em centímetros entre a extremidade inferior da EAV e a marca do paciente é usada como indicador numérico da gravidade da dor ${ }^{(4)}$. No entanto, sua aplicação requer pacientes conscientes e cooperativos. Além disso, é ideal que o paciente seja instruído previamente quanto à utilização da mesma. Se o paciente estiver inconsciente ou incapaz de cooperar, manifestações autonômicas, como palidez, lacrimejamento, sudorese, hipertensão, tacjuicardia, midríase, dispnéia, náuseas e vômitos podem refletir sintomatologia dolorosa e o diagnóstico diferencial com outros quadros clínicos impõe-se ${ }^{(5)}$. A agitação e as alterações das expressões faciais são também importantes parâmetros de observação, sendo que estas podem ser avaliadas através de urna escala de faces. A dor deve ser avaliada através de estímulos provocativos tais como inspiração profunda, tosse, deambulação ou movimentação ativa e sempre em intervalos regulares, variáveis de acordo com a proximidade cronológica da agressão. A distinção entre dor "de repouso" e dor "à movimentação" tem implicações na abordagem terapêutica da dor. Além das questões do conforto e da humanização na UTI, a analgesia visa capacitar o paciente para a realização de fisioterapia, deambulação fora do leito, higiene pessoal diária, cuidado de curativos e alimentação. Além da EAV. podem ainda ser empregada a escala numérica e verbal $(\mathrm{O}=$ ausência de dor; $1 \mathrm{O}$ dor imaginável $)$ ou descritiva e verbal $(\mathrm{O}=$ dor ausente; $1=$ dor leve; $2=$ dor moderada; $3=$ dor intensa), que dependem apenas de comunicação verbal. A EAV é sensível a pro- cedimentos farmacológicos e não farmacológicos, que alteram a sensibilidade à dor, e se correlaciona bastante com a dor medida nas escalas de gradação verbais e numéricas. As instruções para os pacientes graduarem a intensidade da dor ou percentual de alívio da dor, usando a EAV (p.ex., depois de tratamento destinado a reduzir a dor) pode introduzir uma tendenciosidade desnecessária (expectativa de alteração e dependência de memória), a qual reduz a validade da medida. É possível obter uma medida mais correta da alteração, pedindo-se aos pacientes para graduarem a intensidade absoluta da dor, em diferentes mo mentos (p.ex., antes e depois da intervenção) ${ }^{(4)}$. Há, portanto, uma necessidade de escolher adequada mente o método que melhor permita avaliar a dor cio paciente em um determinado momento. A seguir, assume importância o valor referido na escala, a partir do qual será instituída a terapia antálgica. Este valor é variável entre as equipes, porém admite-se, arbitrariamente, o valor " 3 " na EAV ou o relato de dor moderada (escala descritiva verbal) para iniciar a terapia ou para modificá-la, caso um plano terapêutico já esteja estabelecido ${ }^{(4)}$.

\section{MODALIDADES DE ANALGESIA}

\subsection{Analgesia Periférica}

O uso de antiinfiamatórios não esteróides (AINEs) interfere na produção de prostaglandinas, que podem produzir hiperalgesia. Ainda que limitada por efeitos adversos, a vantagem da sua utilização, dos AINEs, é a ausência de depressão do sistema nervoso, cardiovascuiar e respiratório. Além disso, os antiinflamatórios reduzem a necessidade de uso de opióides e, também, como estes, exibem um "efeito teto", ou seja, um aumento na dosagem provoca apenas um aumento na incidência de efeitos colaterais, sem aumento da eficácia analgésica. Entre os efeitos colaterais a serem lembrados estão a influência sobre a hemóstase (inibição reversível sobre a tromboxana A2), o risco de lesão da mucosa gástrica (risco moderado se o tratamento for inferior a 5 dias), alteração da função renal (aumento da resistêlicia vascular renal, hipercalemia, retenção hidrossalina, risco elevado em pacientes hipovolêmicos) e o risco de indução de broncoespasmo, em pacientes asmáticos. Assim, podese afirmar que estejam formalmente contra-indicados nas seguintes situações: insuficiência renal, insuficiência hepática, insuficiência cardíaca, tratamento com 
diurético ou inibidor da enzima de conversão, patologia digestiva com risco hemorrágico, asma de componente alérgico, alergia conhecida ao acenes, terceiro trimestre da gestação ${ }^{(6,7,8)}$. A dipirona (metamizol) e o paracetamol (acetaminofen) são substâncias analgésicas, desprovidas de atividade antiinflamatória.

A infiltração local de anestésicos locais (AL) pode diminuir a necessidade de analgésicos por vários dias, provavelmente devido ao bloqueio dos impulsos nociceptivos aferentes, e diminuir a excitabilidade dos neurônios da medula ${ }^{(8)}$.

O bloqueio intermitente dos nervos intercostais pode ser empregado para a analgesia de lesões torácicas e para o tratamento da dor pós-operatória. Indica-se esta técnica no tratamento da dor torácica ou abdominal alta unilateral, por exemplo, o paciente com tosse ineficaz, em conseqüência de fraturas de arcos costais. As desvantagens incluem a necessidade de injeções repetidas, o risco de pneumotórax $(<0.1 \%)$ e reações tóxicas sistêmicas aos AL, se forem utiliza das quantidades excessivas. Pode ser realizado sob visão direta, durante uma toracotomia. O bloqueio intercostal por via paravertebral ou analgesia interpieural também são alternativas à técnica por injeção múltipla e têm como principal vantagem à possibilidade de introdução de um cateter, o que permite a administração intermitente de AL. Esta última desperta interes se nos casos em que pode haver suspeita de lesões vertebrais graves, com fraturas de $\operatorname{arcos} \operatorname{costais}{ }^{(8)}$.

O bloqueio do plexo braquial por injeção única ou infusão contínua, com cateter promove analgesia prolongada, que permite a mobilização para a fisioterapia respiratória e outras atividades que seriam limitadas pela dor, no membro superior. O bloqueio contínuo do nervo femoral pode beneficiar pacientes com dor no joelho, coxa, como nas fraturas de fêmur ${ }^{(8)}$.

\subsection{Analgesia medular}

A estimulação nervosa elétrica transcutânea (TENS, no inglês) é um método que emprega dois eletrodos, aplicados superficialmente à pele, capazes de gerar urna corrente elétrica contínua de baixa intensidade, que parece eficaz, quando associado a outros métodos de analgesia. O TENS revelou uma tendência à redução de náuseas e vômitos, íleo adinâmico e atelectasia pós-operatória ${ }^{(8)}$.

A transmissão da informação nociceptiva até os centros superiores (supra-espinhais) pode ser bloqueada ou modulada na raiz nervosa ou na medula espi- nhal com AL ou por interação de opióides, com seus receptores localizados no corno dorsal da medula ${ }^{(1,2)}$. Doses intratecais (subaracnóideas) únicas de opióides (morfina ou fentanil) têm sido administradas no momento da punção lombar para proporcionar analgesia pós-operatória por até vinte e quatro (24) horas (morfina). Doses entre 3 a $7 \mu \mathrm{g} / \mathrm{kg}$ de morfina podem ser, assim, administradas no líquor, por ocasião da realização da raquianestesia, para o procedimento cirúrgico. No entanto, a duração limitada da analgesia com morfina intratecal torna preferível a analgesia epidural contínua com opióides. Além disso, a incidência de efeitos colaterais é discretamente maior. Os opióides por via epidural são eficazes em produzir analgesia. A morfina, devido à baixa iipossolubiiidade, é o opióide mais comumente empregado por esta via, tendendo apermanecerem solução, no líquor, em vez de ser absorvida pelas estruturas da medula ou pela circulação. Existe uma migração liquórica rostral, muito maior da morfina do que a observada com outros opióides mais lipossolúveis, como o fentanil, o qual é prontamente absorvido pelas estruturas próximas. ao local da injeção. A morfina é administrada no espaço penduraI em injeções únicas de 2 a $5 \mathrm{mg}$ ou infusões contínuas de 2 a $4 \mathrm{mg} / 24$ horas. Injetada diretamente no líquor, requer dosagens de $0,2 \mathrm{mg}$

A administração de opióides por via espinhal (intratecal ou penduraI) pode provocar alguns efeitos colaterais, sendo o mais temido a depressão respira tória ( 0.6 a $0.9 \%$ com a morfina). Outros efeitos são náuseas e vômitos, prurido, retenção uninánia e sonolência. Os momentos de maior incidência de depressão respiratória ocorrem em urna a duas horas da administração da morfina e outro, seis (6) a doze (12) horas depois. A maioria dos pacientes apresenta uma sedação importante, precedendo a depressão respira tória, o que constitui o prinleiro sinal da depressão respiratória. O planejamento da dose, baseado na posição do cateter (lombar ou torácico), local da cirurgia e idade do paciente pode evitar a depressão respira tória. A depressão respiratória grave deve ser tratada com naloxona (Narcan ${ }^{\circledR}$ ), em doses de 0.1 a $0.2 \mathrm{mg}$, por via intravenosa, ou 0.4 a $0.8 \mathrm{mg}$, por via intramuscular. Pode ser iniciada urna infusão contínua de $5 \mu \mathrm{g} / \mathrm{kg} / \mathrm{h}$, sendo que doses maiores que $5 \mu \mathrm{g} / \mathrm{h}$ podem re verter parcialmente o efeito analgésico. Entre os efeitos colaterais da naloxona, destacam-se a hipertensão, a taquicardia, o edema agudo de pulmão, a abstinência aguda, a fibrilação ventricular, os tremores e as convulsões ${ }^{(5)}$. 
A analgesia pode ser obtida com a infusão contínua de AL, por via epidural. No entanto, recomendase um nível inicial adequado de analgesia, antes do início da infusão contínua. O emprego da bupivacaína (Marcaína® ${ }^{\circledR}$ Neocaína ${ }^{\circledR}$ ), e mais recentemente da ropivacaína (Naropin $\left.{ }^{\circledR}\right)$, é preferível, em virtude do mínimo efeito de bloqueio motor que tais drogas pro movem, quando injetadas em baixas concentrações, no espaço pendurai. $\mathrm{O}$ volume inicial a ser administrado depende do local (dermátomo) no qual se deseja promover a analgesia e da localização da extremidade distal do cateter epidural. A dose inicial padronizada seria de 6 a $8 \mathrm{rnl} / \mathrm{h}$ de bupivacaína a $0.125 \%$ (sem adrenalina), administrados em bomba de infusão ou de PCA (patient controlled analgesia). Para reduzir a eventualidade de taquifilaxia, aumenta-se a infusão com incrementos de $2 \mathrm{~mL} / \mathrm{h}$, até o máximo de 14 a $16 \mathrm{~mL} /$ h. Se a analgesia for inadequada, no volume máximo, a concentração de bupivacaína é aumentada para 0.25 $\%$ e a velocidade de infusão reduzida para $8 \mathrm{~mL} / \mathrm{h}$. A dose máxima infundida não deve ex ceder $1.5 \mathrm{mg} / \mathrm{kg} /$ $\mathrm{h}$, porém, cada incremento da velo cidade de infusão deve ser precedido de um reforço do bloqueio, como no início, para que se obtenha analgesia adequada. $\mathrm{Na}$ concentração de $0.2 \%$ (recomendada para analgesia), a ropivacaína promove bloqueio motor menos intenso que a bupivacaína. A ropivacaína possui cardio e neurotoxicidades menos acentuadas em relação à bupivacaína. A hipotensão arterial secundária ao bloqueio simpático espinhal é uma desvantagem desta técnica. No entanto, é facilmente controlável com infusão de um volume líquido adequado, ou pela administração de vasopressores tais como a efedrina (bolus de 5 a $10 \mathrm{mg}$ EV) ou fenilefri na (bolus de 50 a $100 \mu \mathrm{g}$ EV). A associação de AL e opióides melhora a qualidade da analgesia e permite o emprego de soluções em concentrações mais reduzi das de AL (bupivacaína $0.0625 \%$ ). A combinação de ambos os tipos de medicação, em baixas doses, proporciona o benefício de cada uma, sem a severidade dos efeitos colaterais observados quando são em pregadas isoladamente. Entre as possíveis indicações desta técnica, podemos citar cirurgias do andar superior do abdômen, toracotomias, esternotomias, dor anginosa (mesmo aquelas refratárias à terapia com nitratos $)^{(9,10,11)}$, pancreatites, dor isquêmica em extremidades ${ }^{(8,12)}$. (Tabela 1).

A clonidina (Clonidin $\left.{ }^{\circledR}\right)$, administrada por via espinhal ou sistêmica, potencializa o efeito analgésico tanto de opióides quanto de $\mathrm{AL}^{(13,14)}$. Empregada isoladamente, são necessárias doses superiores a 300 pg para produzir analgesia, as quais provocam bradicardia, hipotensão e sedação. A clonidina tem sido empregada, alternativamente, por via sistêmica, para o tratamento dos sintomas associados à síndrome de abstinência, provocada por opióides, álcool e cocaína.

Tabela I - Dose sugerida de opioides espinhais para dor pós-operatória

\begin{tabular}{lllrr}
\hline Droga & Dose única & Infusão & Inicio $(\mathrm{min})$ & Duração $(\mathrm{h})$ \\
\hline Epidural & & & & \\
- Morfina & $1-5 \mathrm{mg}$ & $0.1-1.0 \mathrm{mg} / \mathrm{h}$ & 30 & $6-24$ \\
- Meperidina & $20-150 \mathrm{mg}$ & $2-20 \mathrm{mg} / \mathrm{h}$ & 5 & $6-8$ \\
- Fentanil & $25-150 \mu \mathrm{g}$ & $25-100 \mu \mathrm{g} / \mathrm{h}$ & 5 & $3-6$ \\
- Sufentanil & $10-60 \mathrm{pg}$ & $10-50 \mu \mathrm{g} / \mathrm{h}$ & 5 & $2-4$ \\
& & & & \\
Intratecal & & & 15 & $8-24$ \\
- Morfina & $0.1-0.3 \mathrm{mg}$ & - & 5 & $3-6$ \\
- Fentanil & $5-25 \mu \mathrm{g}$ & - & & \\
\hline
\end{tabular}




\subsection{Analgesia Sistêmica}

A infusão intravenosa contínua, de um opióide é uma técnica preferível à administração intermitente, em "bolus". Deve-se acelerar a administração inicial sistêmica para atingir uma concentração analgésica eficaz mínima (CAEM), em um período curto de tempo. A administração contínua de opióide, sem essa dose inicial, em "bolus", não resulta na obtenção da CAEM por um período de, pelo menos, três meias- vidas de eliminação ( $\left.\mathrm{t} 1 \frac{2}{2} \beta\right)$. Após essa dose inicial, um nível sérico, terapêutico, de opióide pode ser obtido por infusão contínua.

A analgesia controlada pelo paciente (do inglês patient controlled analgesia ou PCA) é uma técnica para administração intravenosa de pequenas do ses de opióide ou de qualquer outra droga analgésica, de acordo com a necessidade do paciente. E reduzido o risco de sobredosagem com a PCA, pois os pacientes tendem a quantificarem - se na faixa terapêutica. A PCA pode ser empregada pelas vias intravenosa, subcutânea, epidúral, sublingual e bucal. Existem vá rias maneiras de se administrar uma PCA, porém a administração sob demanda é a mais comum, ou seja, após atingir a CAEM, através da titulação de uma dose inicial em "bolus", uma determinada dose é ad ministrada intermitentemente pelo paciente. Uma infusão basal de velocidade constante pode ser usada para complementar as necessidades do paciente (in fusão constante e administração sob demanda). Para cada modalidade básica de PCA, existem variáveis básicas: dose inicial, dose de demanda, intervalo míni mo, velocidades de infusão basal e limites de uma e quatro horas. A dose inicial é a quantidade de analgésico empregada para obter analgesia. A dose de demanda é a quantidade de analgésico que o paciente recebe por auto-administração quando percebe a necessidade de mais analgésico. O intervalo mínimo é o período de tempo entre solicitações do paciente, durante o qual a bomba infusora não administrará mais analgésicos, ainda que o paciente assim o solicite. A PCA pode ser empregada para a administração de AL ou sua associação com opióides, pela via epidural. A PCA também requer um paciente consciente e cooperativo para seu êxito (Tabela II).

Vale ressaltar que a dor é mais intensa nas horas que seguem o trauma ou a cirurgia, reduzindo-se, progressivamente, nos dias consecutivos. É muito mais intensa nos procedimentos torácicos ou abdominais altos e um pouco menor nos procedimentos do abdômen inferior, extremidades e procedimentos sobre a superfície cutânea. Assim, parece lógico, administrar-se precocemente a associação de AINEs com opióides potentes $($ meperidina $>$ nalbufina $=$ morfina $>$ alfentanil $>$ fentanil > sufentanil, em ordem crescente de potência) e os AL. Particularmente, estes últimos são eficazes em abolir a dor à movimentação, tarefa impossível, quando da utilização exclusiva de opióides. A medida que a dor se reduz, os opióides potentes são substituídos por opióides fracos (tramadol. codeína, propoxifeno) e, finalmente, são suprimidos. A meperidina ou petidina (Dolantina ${ }^{\circledR}$, Dolosal $\AA$ ) merece um pouco mais de atenção devido ao seu potencial de toxicidade e interação medicamentosa. A meperidina é uma droga dez (10) vezes menos potente que a mor fina, que, após metabolização hepática, produz a normeperidina, substância potencialmente tóxica, capaz de causar mioclonias e convulsões, principalmente em pacientes portadores de insuficiência renal e idosos. A meperidina tem interação medicamentosa com os inibidores da monoaminooxidase (IMAO), provocando hipertermia, hipertensão, taquicardia, hipotensão, rigidez torácica, convulsões e coma. Portanto, a sua administração prolongada é desaconselhada $^{(3,6)}$. A morfina possui um efeito mais prolongado em pacientes renais crônicos, pois seus metabólitos ativos possuem excreção renal.

\section{SEDAÇÃO}

Em terapia intensiva, os principais objetivos da sedação incluem reduzir a resistência à ventilação mecânica, tratamento de distúrbios psiquiátricos ou problemas relacionados à abstinência de substâncias de abuso, restauração da temperatura corpórea, redução da ansiedade, facilitação do sono e redução do metabolismo ${ }^{(3,6)}$. Em casos de traumatismo craniano, o objetivo da sedação pode incluir a indução do coma,
Tabela II - Doses sugeridas de opioides para uso na analgesia controlada pelo paciente (PCA)

\begin{tabular}{lcc}
\hline Droga (conceritraçao) & $\begin{array}{c}\text { Dose de } \\
\text { demanda }\end{array}$ & $\begin{array}{c}\text { Intervalo minimo } \\
\text { (min.) }\end{array}$ \\
\hline Fentanil $(10 \mu \mathrm{glmL})$ & $10-20 \mu \mathrm{g}$ & $5-10$ \\
Meperidina $(10 \mathrm{mg} / \mathrm{mL})$ & $5-30 \mathrm{mg}$ & $5-10$ \\
Morfina $(1 \mathrm{mglmL})$ & $0.5-3.0 \mathrm{mg}$ & $5-10$ \\
Nalbufina $(1 \mathrm{mg} / \mathrm{mL})$ & $1-5 \mathrm{mg}$ & $5-10$ \\
\hline
\end{tabular}


a fim de promover o "silêncio elétrico" (EEC burst supression, do inglês) do cérebro, reduzindo sua necessidade metabólica. Tal procedimento pode estar associado ou não à indução de hipotermia para controlar as necessidades metabólicas neuronais. $\mathrm{O}$ agente sedativo ideal deve possuir propriedades ideais como mínimo efeito depressor dos sistemas respiratório e cardiovascular, não interferência no metabolismo de outras drogas, e possuir vias de eliminação independentes dos mecanismos renal, hepático ou pulmonar, resultando em uma meia-vida de eliminação curta, sem metabólitos ativos ${ }^{(3,6,16)}$.

A sedação pode ser definida entre um simples estado de cooperação, com orientação espaçotemporai e tranqüilidade ou apenas resposta ao comando, podendo incluir ou não a hipnose ${ }^{(3,6,17)}$. Para se avaliar o grau de sedação, empregam-se inúmeras escalas, sendo a mais utilizada aquela proposta por Ramsay. A escala de Ramsay é utilizada como referência para validação de novas técnicas. Recentemente, foi introduzida a análise do índice biespectral do eletroencefalograma corno monitor da profundidade anestésica. Esta técnica guarda uma boa correlação com a escala de Ramsay. No entanto, este recurso ainda requer mais estudos para sua validação como monitor cia profundidade da sedação. A avaliação da sedação ainda se baseia fundamentalmente na observação clínica ${ }^{(3,6)}$.

Entre os agentes farmacológicos disponíveis para a sedação, os opióides são drogas que, além do seu efeito analgésico, promovem sedação importante e são empregados em pacientes requerendo ventilação mecânica, pois inibem a ventilação, bem como o reflexo de tosse. São empregados em associação com os benzodiazepínicos ou com o propofol. O uso prolongado induz à ocorrência de tolerância. Os mais comumente empregados são o fentanil, alfentanil, sufentanil e a morfina. O uso concomitante de opióides reduz a necessidade de outros hipnóticos ${ }^{(17)}$.

Os benzodiazepínicos (BZD) são as drogas mais amplamente utilizadas na UTI. Os BZD possuem excelentes qualidades sedativas hipnóticas e ansiolíticas, associadas à ação anticonvulsivante e relaxante muscular A amnésia constitui-se num efeito colateral desses compostos. Em relação ao sistema cardiovascular, possuem alguns efeitos benéficos, relacionados à redução tanto da pré-quanto da pós-carga, devido à discreta ação simpatolítica. Os BZD diminuem o consumo de oxigênio miocárdico, porém, quando associa dos a doses elevadas de opióides podem produzir uma importante depressão miocárdica, sobretudo nos pa cientes que apresentam má função ventricular. As cio sagens devem ser adequadamente tituladas em pacientes hipovolêmicos. Os BZD produzem redução do fluxo sanguíneo cerebral (FSC), de maneira acoplada à redução do consumo de oxigênio cerebral. Desta maneira, esses compostos são úteis na redução da pressão intracraniana, à condição que hipotensão seja evitada de maneira a garantir uma pressão adequada de perfusão cerebral $(\mathrm{PPC}=\mathrm{PAM}-\mathrm{PIC})$. Em pacientes ventilando espontaneamente, principalmente em idosos e pneumopatas, os BZD reduzem a resposta ventilatória reflexa induzida pela hipoxemia e hipercapnia. Os dois principais BZD disponíveis para administração intravenosa no Brasil são o midazolam (Dormonid ${ }^{\circledR}$ ) e o diazeparn (Valium ${ }^{\circledR}$ ). Ambos são dependentes do mecanismo de glicuronidação hepática para serem metabolizados e, portanto, seus efeitos são prolongados nos pacientes com insuficiência hepática. O metabolismo do diazepam gera o n-desmetil diazepam que, como o próprio composto original, possui uma meia-vida de eliminação prolongada (> 20 horas). O efeito do diazepam é tão mais prolongado quanto for a duração da sedação. $\mathrm{O}$ midazolain possui uma meia-vida de eliminação de duas (2) a quatro (4) horas e não possui metabólitos ativos, portanto encontra um perfil mais adaptado à infusão contínua, por períodos curtos ou prolongados. Devido à extensa ligação protéica (96\%), os efeitos sedativos podem ser importantes em pacientes hipoproteinêmicos e hipoalbuminêmicos, devido à maior fração de droga livre no plasma ${ }^{(16)}$.

O flumazenil (Lanexat ${ }^{\circledR)}$ é o antagonista competitivo dos receptores diazepínicos. Em UTI, pode ser empregado em doses crescentes de $0.3 \mathrm{mg}$, até uma dose total de $2 \mathrm{mg}$, em intervalos de um (1) mi nuto. O flumazenil permite antagonizar os efeitos hipnóticos e sedativos dos BZD, e os efeitos adversos paradoxais, como a agitação. Além disso, o flumazenil permite o diagnóstico e o tratamento de uma intoxicação por BZD ou pode ser auxiliar no diagnóstico etiológico do coma. Está contra-indicado em pacientes alérgicos aos BZD, em pacientes em uso crônico de BZD ou naqueles que os utilizam para controle da epilepsia. Devido à meia-vida de eliminação ser mais curta do que a dos BZD, existe o risco de ressedação. A reversão dos efeitos dos BZD pode promover elevação da PIC e descompensação cardiovascular.

O propofol (Diprivan () é uma droga anestésica, capaz de induzir desde a sedação até uma hipnose 
profunda, com características do burst supression do $\mathrm{EEG}^{(18)}$. Em razão do seu metabolismo elevado, possui curta meia-vida de eliminação. No entanto, após uma infusão prolongada (de duração média de oitenta e cinco (85) horas) ocorre um aumento na meia-vida de eliminação que não compromete o despertar dos pacientes, sendo que as concentrações plasmáticas se reduzem em $50 \%$, após dez (10) minutos da interrupção da infusão(17,18). Em comparação com o midazolam, o propofol está associado com mais rápida recuperação dos efeitos clínicos e eletroencefalográficos. As condições clínicas são semelhantes, quando se associam a opióides do tipo propofol ou midazo Iam. Pode ser utilizado no traumatismo craniano, desde de que mantida a PPC (> $60 \mathrm{mmHg}$ ), para uma redução da PIC inferior a $10 \mathrm{mmHg}$. Após cirurgia cardíaca, mostra-se interessante na sedação por curta duração para manutenção da assistência ventilatória. No entanto, uma redução de 15 a $20 \%$ da pres são arterial média pode ser observada após urna dose em "bolus", variando entre 0.24 e $1 \mathrm{mg} / \mathrm{kg}$. A freqüência cardíaca pode ser reduzida devido à inibição do barorreflexo, condição vantajosa nos pacientes coronariopatas. Outras indicações para o uso do propofol incluem a redução do espasmo tetânico, broncodilatação, em pacientes pneumopatas, tratamento do estado de mal epiléptico, refratário a outras medicações, redução de náuseas e vômitos e do prurido, e cardioversão. O propofol é urna droga de aplicação segura nos pacientes susceptíveis à hipertermia maligna ${ }^{(3,6,17,18,19)}$.

Embora o propofol tenha sido usado com sucesso para sedação de crianças na UTI, há relatos de seqüelas neurológicas, após a sua interrupção, e de ocorrência de acidose metabólica, durante a infusão na vigência de infecção das vias aéreas superiores $^{(20,21)}$. O propofol pode induzir hiperlipidemia, quando infundido por tempo prolongado e doses elevadas. Existem relatos de casos de pancreatite aguda associados ao seu uso. Pode existir urna tendência a esverdeamento do cabelo e da urina, e coloração esbraquiçada da urina devido à lipidúria ${ }^{(17)}$.

Os barbitíricos ainda são utilizados, porém com freqüência cada vez menor, devido à extensão da meiavida de eliminação após infusão, que resulta em acúmulo nos tecidos periféricos (muscular e sobretudo gorduroso). O tiopental (Thionembutal ®) ainda é o mais empregado. Utilizam-se doses iniciais de 2-4 $\mathrm{mg} / \mathrm{kg}$, seguidas de infusão $30-80 \mu \mathrm{g} / \mathrm{kg} / \mathrm{min}$. É bastante utilizado na sedação de pacientes neurocirúrgicos ou traumatizados cranianos $^{(6)}$. (Tabela III).

\section{BLOQUEIO NEUROMUSCULAR (CURARIZAÇÃO)}

A curarização resulta da ação de drogas (cura res) na junção neuromuscular, que, atuando, na maioria das vezes, corno antagonistas competitivos da acetilcolina, impedem a ligação desta aos receptores nicotínicos, localizados na porção pós-sináptica da junção. Clinicamente, se expressa pela paralisia muscular flácida ou pela fraqueza muscular generalizada.

Há algumas situações nas quais os curares es tão indicados, mas não são indispensáveis. Tais situações são a entubação traqueal, reaquecimento pósoperatório com tremores, presença de anastomoses vasculares delicadas, a necessidade de proteção de feridas sob tensão, anastomose traqueal, pressão intracraniana elevada, inserção de cateteres vasculares invasivos, em pacientes agitados, facilitação da ventilação mecânica, controle das convulsões, durante o estado de mal epiléptico e o controle dos espasmos tetânicos. A necessidade de curares pode ser bastante reduzida ou mesmo abolida, se sedação e analgesia adequadas forem realizadas

Raramente um paciente sob ventilação mecânica requer curarização. A curarização está indicada em pacientes com baixa complacência torácica ou pulmonar, naqueles desadaptados à ventilação mecânica e naqueles nos quais existe um risco de barotrauma devido às pressões elevadas das vias aéreas. Nas modalidades de ventilação com relação I:E invertida e naquelas com geração de altos volumes por minuto, a curarização pode ser indicada.

A succinilcolina (Quelicin ${ }^{\circledR}$ ) é usada na UTI para entubação traqueal, principalmente quando o tônus muscular da mandíbula impede a laringoscopia. Adose é de $1-2 \mathrm{mg} / \mathrm{kg}$, intravenosa. E útil em pacientes ditos de "estômago cheio", nos quais a entubação

\begin{tabular}{|c|c|c|}
\hline Droga & "Bolus" ( $\mu \mathrm{g} / \mathrm{kg})$ & $\begin{array}{r}\text { Manutenção } \\
(\mu \mathrm{g} / \mathrm{kg} / \mathrm{min})\end{array}$ \\
\hline Alfentanil & $10-25$ & $0.25-1$ \\
\hline Fentanil & $1-3$ & $0.01-0.03$ \\
\hline Sufentanil & $0.1-0.3$ & $0.01-0.05$ \\
\hline Mldazolam & $25-100$ & $0.25-1$ \\
\hline Propofol & $250-1000$ & $10-50$ \\
\hline
\end{tabular}


seqüêncial rápida, com manobra de compressão sobre a cartilagem cricóide (manobra de Sellick) está indicada. Provoca fasciculações musculares devido a uma despolarização persistente, responsável pela elevação do potássio sérico de $0.5-1 \mathrm{mmol} / \mathrm{L}$ e que pode gerar arritmias cardíacas. Esta hipercalemia é mais intensa após queimaduras, paraplegia ou hemiplegia, trauma muscular, e por lesão do neurônio motor superior, conseqüência de trauma ou acidente vascular cerebral. A succinilcolina aumenta a pressão intragástrica, podendo causar regurgitação e aspiração, além de ser contra-indicada em pacientes susceptíveis de hipertermia maligna.

O pancurônio (Pavulon $®)$ é o curare mais empregado em UTI. É um curare de duração de ação entre quarenta e cinco e sessenta (45-60) minutos e dependente de mecanismos renais $(70 \%)$ e hepáticos (30\%) para sua eliminação, acumulando-se nos pacientes com insuficiência renal ou hepática, quando administrado em doses mais elevadas. Possui um efeito vagolítico que determina hipertensão e taquicardia.

O atracúrio (Tracrium ${ }^{\circledR}$ ) é um curare de duração intermediária, ao redor de vinte (20) minutos. E eliminado por hidrólise espontânea plasmática, independente dos mecanismos renais e hepáticos. Os efeitos cumulativos estão praticamente ausentes e, por isso, está aconselhado para utilização sob infusão contínua. Pode liberar histarnina, principalmente quando administrado rapidamente.

O vecurônio (Norcuron ${ }^{\circledR}$ ) é um curare de duração intermediária (trinta (30) minutos) análogo ao pancurônio, porém com menos efeitos sobre o sistema cardiovascular. É primariamente metabolizado pelo fígado, e dependente dos rins para a excreção. A dosagem deve ser reduzida na insuficiência hepática e renal. Adapta-se à infusão contínua, embora produza um metabólito ativo que possui metade da potência do composto original.
O rocurônio (Esmeron $®$ ) em doses de $0.6 \mathrm{mg} / \mathrm{kg}$, produz boas condições de entubação entre sessenta e noventa (60-90) segundos e duração de efeito prolongado (quarenta (40) minutos). Outros curares como o doxacúrio, pipecurônio e mivacúrio são curares com pouca experiência de utilização em UTI (Tabela IV).

O bloqueio neuromuscular pode ser antagonizado (exceto no caso da succinilcolina) por drogas anticolinesterásicas. Em nosso meio, o agente mais empregado é a neostigmina (Prostigmine ${ }^{\circledR}$ ) na dosagem de $0.05 \mathrm{mg} / \mathrm{kg}$. A administração concomitante de atropina $(0.01 \mathrm{mg} / \mathrm{kg})$ é preconizada com a finalidade de atenuar a estimulação sobre os receptores periféricos muscarínicos, reduzindo, principalmente, a intensa bradicardia, determinada por estes agentes. A intensidade do bloqueio neuromuscular pode ser monitorizada através da estimulação do nervo periférico e as dosagens podem ser, assim, ajustadas para uma intensidade mínima de depressão da atividade muscular. No entanto, a monitorização com o estimulador de nervo periférico possui um entendimento mais complexo que foge ao objetivo desta revisão.

O bloqueio neuromuscular, por tempo prolongado, pode induzir a uma disfunção neuromuscular, caracterizada por fraqueza muscular generalizada, tetraparesia, arreflexia, progredindo até a paralisia flácida, persistente por dias ou meses. Em alguns pacientes, uma associação entre curare e corticoterapia, em altas dosagens (mal asmático, transplantado hepático) foi observada. Deve ser diferenciada da polineuropatia da UTI, que ocorre por ocasião da sepse, na ausência da administração de qualquer curare. Cabe ainda lembrar que inúmeras drogas potencializam o efeito dos curares, tais como os aminoglicosídeos, tetraciclinas, anestésicos locais, fenitoína, propranolol, trimetafam, glicosídeos cardíacos, cloroquina, catecolaminas e diuréticos ${ }^{(23)}$.

Tabela IV - Agentes Curarizantes mais empregados em terapia intensiva

\begin{tabular}{lccccl}
\hline Droga & "Bolus"mg/kg & $\begin{array}{c}\text { Manutenção } \\
\mathrm{mg} / \mathrm{kg} / \mathrm{min}\end{array}$ & $\begin{array}{c}\text { Efeito } \\
\text { minutos }\end{array}$ & $\begin{array}{c}\text { Duração } \\
\text { minutos }\end{array}$ & Complicaçao \\
\hline Succinilcolina & $1-2$ & não recomendada & $0.5-1$ & $5-10$ & ação vagolitica \\
Pancuronio & 0.1 & $0.3-0.5$ & 3 & $45-60$ & hipertensão taquicardia \\
Atracurio & 0.5 & $3-10$ & $1.5-2$ & 20 & liberação histamina \\
Vecuronio & 0.1 & $1-2$ & $2-3$ & $25-30$ & nenhum \\
Rocuronio & 0.6 & $9-12$ & $1-1.5$ & $20-40$ & nenhum \\
Mivacurio & 0.15 & $3-10$ & 2 & $15-20$ & liberação histamina \\
\hline
\end{tabular}


SLULLITEL A \& SOUSA AM. Analgesia, sedation and neuromuscular blockade in the ICU. Medicina, Ribeirão Preto, 31: 507-516, oct./dec. 1998.

ABSTRACT: Pain is a major symptom which is often found in critically iii patients. Adequate management of this condition is implicated not only with humanization process in the ICU but may also to improve outcome and reduce hospital costs. Importance of measuring daily pain scores by the ICU team, as well as the available techniques of providing a good pain relief are pointed out. Exposition to a noxious environment which includes pain, noise, tracheal suctioning, sensory overload or sleep deprivation may require the use of drugs to promote sedation in order to control anger and mental stress. Furthermore, some clinical conditions, such as mechanical ventilation, may require sedation for its success. Some clinical aspects of neuromuscular blocking agents and their uses in the ICU are also reviewed.

UNITERMS: Analgesia. Neuromuscular Blocking Agents. Conscious Sedation. Intensive Care Units.

\section{REFERÊNCIAS BIBLIOGRÁFICAS}

1 - BONICA JJ. Definitions and taxonomy of pain .In: BONICA JJ., ed The management of pain. $2^{\text {nd }}$ ed. Lea \& Febiger, Phyladelphia, cap. 2 , p.18-19, 1990.

2- BONICAJJ. Anatomic and physiologic basis of nociception and pain. In: BONICA JJ., ed. The management of pain. $2^{\text {nd }}$ ed., Lea \& Febiger, Philadelphia, cap.3, p. 28-94, 1990.

3- TERZI R \& AMARAL JLG. Consenso brasileiro sobre analgesia, sedação e bloqueio neuromuscular em terapia intensiva Clin Bras Med Intensiva 2: 241-254, 1996.

4 - KATZ J \& MELZACK R. Mensuração da dor. Clín Anestesiol Am Norte 2: 225-242, 1992.

5- MURRAY MJ. Problemas de dor na UTI. Clín Ter Intensiva 2: 247-265, 1990.

6- SHAPIRO BA et al. Practice parameters for intravenous analgesia and sedation for adult patients in the intensive care unit: An executive summary. Crit Care Med 23: 1596-1600, 1995.

7- SOUTER AJ. Nonsteroidal anti-infiammatory drugs. In. WHITE PF. ed. Textbook of intravenous anesthesia. Williams \& Wilkins, Baltimore, cap. 12, p. 247-257, 1997.

8 - LEE VC. Modalidades de medicações não-narcóticas para o tratamento da dor aguda. Clín Ter Intensiva 2: 465-495, 1990.

9 - OLAUSSON K ei ai. Anti-ischemic and anti-anginai effects tionai medical therapy in the treatment of severe refractory unstable angina pectoris. Circulation 96: 2178-2182, 1997.

10- RAMAMURTHY S. Thoracic epidurai nerve block. In: Waldman SD \& Winnie AP, ed. Interventional pain management. WB. Saunders, Philadelphia, cap. 27, p. 304-309, 1996.

11 - MEISSNER A et al. Thoracic epidural anesthesia and the patient with heart disease: Benefits, risks, and controversies. Anesth Analg 85: 517-528, 1997.

12 - RAJ PP. Continuous epidural infusion and patient-controlled epidural analgesia in the management of pain. in: WaldmanSD \& Winnie AP ed. Interventional pain management. W.B. Saunders, Philadelphia, cap.31, p. 333-338, 1996.
13- BADNER NH. Agentes administrados por via epidural para analgesia pós-operatória. Clín Anestesiol Am Norte 2: 317-333, 1992

14- EISENACH J. Analgesic drug classes in the management of clinical pain: a In: Yaksh TL. et ai. ed. Anesthesia: biologic foundations. Lippincott-Raven, Philadelphia, cap. 59, p. 935-942, 1997.

15- FERRANTE MH. Analgesia controlada pelo paciente. Clin Anestesiol Am Norte 2: 283-294, 1992.

16- HOWIE MB. Use of intravenous techniques in critically iii patients. In: WHITE PF. ed. Textbook of intravenous anesthesia. Wiliiams \& Wilkins, Baltimore, cap. 25, p. 503$513,1997$.

17- MIRENDA J \& BROYLES G. Propofol as used tor sedation in the ICU. Chest 108: 539-548, 1995.

18- WHITE PF. Propofol. In: WHITE PF ed. Textbook of intravenous anesthesia. Wiiliams \& Wilkins, Baltimore, cap. 7, p. 111-152 1997

19- BORGEAT A ei al. The nonhypnotic therapeutic apphcations of propofol. Anesthesiology 80: 642-656, 1994.

20 - MARTIN PH et al. Metabolic and hemodynamic effects of infusion 01 propofol for long-term sedation of children undergoing intensive care. Br J Anaesth 79: 276-279, 1997.

21 - HATCH DJ. Propofol in paediatric intensive care. Br J Anaesth 79: 274-275, 1997.

22- HUNTER JM. New Neuromuscular biocking drugs. N Engi J Med 332: 1691-1699, 1995.

23 - SHAPIRO BA et al. Practice parameters for sustained neuromuscular blockade in the aduit critically ill patient: an executive summary. Crit Care Med 23: 1601-1605, 1995.

Recebido para publicação em 04/03/98

Aprovado para publicação em 07/1 0/98 